\title{
Forgetting of a CS attribute in a conditioned suppression paradigm
}

\author{
DAVID A. THOMAS and DAVID C. RICCIO \\ Kent State University, Kent, Ohio 44240
}

\begin{abstract}
Although a number of studies have demonstrated nearly complete retention of fear in a conditioned suppression task, they provide little information about the nature of the memory for the CS. The purpose of the present experiment was to investigate the retention of an attribute of a tonal CS that had been paired with shock and was thus capable of eliciting a conditioned emotional response (CER) in rats. The Kamin blocking effect was utilized to detect changes in the memory of CS attributes. Either 1 or 21 days following conditioning to a tone, separate groups of rats received compound conditioning in which either the original or a novel tone was combined with a light. Subsequent measurement of suppression to the added element (light) indicated that only the original CS produced blocking at the short delay, but that both original and novel tones resulted in blocking at the 21-day interval. This increase in the extent of blocking suggests that memory for specific attributes of the CS does diminish as a function of time.
\end{abstract}

Compared with other areas of memory research, retention of conditioned suppression (CER) has received relatively little attention. One reason may be that, typically, little forgetting occurs in the CER paradigm. For example, Hoffman, Selekman, and Jensen (1966) reported nearly complete retention of CER by pigeons over a 30 -month interval. A subsequent study by Gleitman and Holmes (1967), designed to correct certain methodological problems in the Hoffman experiment, obtained quite similar results. Retention of an incompletely learned CER in rats did not differ between groups tested after intervals of 1 or 90 days (Gleitman \& Holmes, 1967). Furthermore, several other studies have found little, if any, forgetting of conditioned suppression in adult rats (Campbell \& Campbell, 1962; Coulter, Collier, \& Campbell, 1976; Frieman, Warner, \& Riccio, 1970; Snedden, Spevack, \& Thompson, 1971).

Although these studies clearly demonstrate strong retention of the CS-UCS contingency (fear), they provide little information about the nature of the memory for the CS. In all of these tests of CER retention, the animals were exposed to either the training stimulus only (e.g., Gleitman \& Holmes, $1967)$ or to a generalization test which included all of the test stimuli (e.g., Hoffman et al., 1966). Spear

\footnotetext{
This article is based on part of a dissertation submitted by the first author to Kent State University, Department of Psychology, 1977. The research was supported by NIMH Grant MH 30223-01 awarded to the second author. An initial report of these data was made at the annual meeting of the Eastern Psychological Association, Boston, 1977. D. A. Thomas, to whom requests for reprints should be sent, is now at Rutgers University, Livingston College, Department of Psychology, New Brunswick, New Jersey 08903 .
}

(1973) has suggested that the memory of any episode may consist of a number of attributes. This analysis would maintain that sufficient cues for retention are available in the single-tone CER test. Perhaps it takes only a weak memory (or minimal attributes) to result in suppression in the single-tone CER test. In a sense, the single-tone CER test may be methodologically too sensitive in that it reflects any above-threshold memory. On the other hand, a within-groups CER design may also be inappropriate for testing retention. Pigeons (Hoffman et al., 1966) or rats (Freiman et al., 1970) tested using a within-groups CER design were provided with comparison test tones, and consequently the resulting steep generalization gradient may have reflected the presence of additional attributes rather than a flawless memory. Whether the CER test (single stimulus or within-groups design) performance is a veridical representation of the training episode remains in doubt. Thus, it is possible that certain attributes or characteristics of stimuli in the training situation are degraded during a retention interval but that such changes do not manifest themselves in terms of changes in conditioned suppression over time in the single-stimulus or the within-groups CER generalization tests.

A recent study by Coulter (Note 1) provides some support for this interpretation. In Coulter's study, rats received fear conditioning and then were tested for suppression either to all of the generalization tones (within groups) or to only one of the various test tones (between groups). This testing was done immediately ( 1 day) or after a delay ( 2 weeks). Only the rats in the between-groups delay conditions showed suppression to all of the test tones. Coulter argued that these animals forgot specific attributes of 
the CS and showed generalized suppression to all of the tones. Rats tested using the within-groups design, either immediately or after a delay, displayed more discrimination, as evidenced by the steeper generalization gradients. This result is consistent with previous reports of CER retention performance. Presumably, they were provided with a sufficient amount of retrieval cues. However, rats tested 1 day after training in the between-groups design were able to discriminate among the test tones, as evidenced by decreasing amounts of suppression to tones that differed from the original training tone. Coulter demonstrated that CS attributes (in this case, frequency of a tone) are subject to retrieval failure which can be compensated for by using a within-groups generalization test (cf. Spear, 1973).

Since the use of a between-groups generalization CER test by Coulter was unique and her results were dependent on that design, replication of the phenomenon seemed desirable in order to show that the effect was reliable and not restricted to her methodology. Another approach to evaluating retention of CS attributes, using a between-groups CER design, would involve new learning at testing. One might ask: to what extent does the previously learned information transfer to a second task? In the present study, the Kamin blocking paradigm (Kamin, 1968) was employed to measure retention of a CS value. Since the typical finding is dependent upon retention of CER, the procedure should be particularly sensitive to deviations in the retention. Kamin (1968) has shown that conditioning to an element of a compound stimulus will be attenuated if conditioning to the other element of the compound stimulus has previously been established. In order to examine retention of stimulus attributes, we took advantage of this phenomenon by manipulating the original element at the time of compound conditioning. We assumed that if rats retained specific information about the original CS, then a shift in its value should no longer produce the blocking effect, as both elements are functionally novel. If, however, forgetting of the CS attributes has occurred, as might be the case after a long retention interval, then a change in some aspect of the CS might not disrupt blocking. That is, if the presentation of $\mathbf{A}^{\prime}$ during compound training cannot be distinguished from the memory of the original $\mathbf{A}$, then blocking of conditioning to the added element should occur.

The general plan of this experiment was as follows: Rats were conditioned to a tone, and either 1 or 21 days later conditioned to a compound stimulus consisting of a light and either the original tone or a different tone. Following this training, all rats were tested for suppression to the added element, i.e., the light. To the extent that the rats forget the original tone, they should perceive the tone of the compound stimulus as if it were the original tone. Accordingly, in this situation, conditioning to the light should be limited. If rats do not forget CS attributes, such as frequency of a tone, the performance of the 21day groups should be consistent with the groups that received the compound training 1 day after conditioning to the tone was completed.

\section{METHOD}

\section{Subjects}

Thirty male Holtzman albino rats, approximately 65 days old at the beginning of experimentation, were housed in individual cages. The rats were reduced to $80 \%$ of their free-feeding weights via water rationing. Food was available ad lib.

\section{Apparatus}

Two operant conditioning chambers, measuring $20.3 \times 22.9 \times$ $\times 19.7 \mathrm{~cm}$ high, were used for the barpress training, fear condition ing, and retention testing. The chambers were housed in sound-attenuating, opaque chests. Exhaust fans, mounted on the back of each chest, further reduced outside noise. Two walls and the ceiling of the chambers were Plexiglas, whereas the remaining two walls were aluminum, including the one which contained a manipulandum, water dipper, and a cue light. The water dipper, which was mounted in the center of the wall $1.9 \mathrm{~cm}$ from the floor, delivered approximately $.1 \mathrm{cc}$ reinforcement. The manipulandum was a $1.9 \times 3.18 \mathrm{~cm}$ bar located $3.8 \mathrm{~cm}$ from the floor and $5.0 \mathrm{~cm}$ to the right of the water dipper. The white, round, jeweled cue light was located $3.8 \mathrm{~cm}$ above the bar. Speakers were located on the ceilings of each chamber, and a $70-\mathrm{dB}$ tone was delivered by a single audio oscillator. A single matched-impedance shock source (Campbell \& Teghtsoonian, 1958) delivered a .5-sec, .5-mA shock, scrambled by a Foringer Model SC -901 shock scrambler, through grid floors. The grids consisted of $1.5-\mathrm{mm}$ stainless steel bars placed $1 \mathrm{~cm}$ apart. All the experimental procedures were controlled by relay equipment in a room adjacent to the experimental room.

\section{Procedure}

On the 1st day, all rats were placed in the operant chambers for 15 min on a CRF schedule. On subsequent days, all sessions were $30 \mathrm{~min}$ long. On Day 2, reinforcement was again delivered for each barpress, but on Day 3 a VI 1-min schedule of reinforcement was instituted and was in effect for the remainder of the experiment.

Acquisition of barpress suppression to a $3,160-\mathrm{Hz}$ tone began on Day 6 for all rats. This tone was presented during the 12 th and the 24 th minute of each session. The tone was $1 \mathrm{~min}$ in duration and was followed immediately by the shock. All rats received 10 tone-shock pairings over five sessions during this initial acquisition phase. At the end of this training, the rats were divided into six groups of five based on their suppression performance.

For three of the groups, the second acquisition phase began the next day. In this second phase, acquisition of suppression to a compound light-tone stimulus was the required response. The tone used in the second phase for one of the groups $($ Day $/ 3,160)$ was the same as that used in the previous acquisition sessions. For the other two groups (Day/1,000 and Day/10,000), however, a novel tone of either $1,000 \mathrm{~Hz}$ or $10,000 \mathrm{~Hz}$ was used as a component of the compound stimulus. The cue light was identical for all three groups. All rats received $101-\mathrm{min}$ presentations of their compound stimulus followed by the previously used shock. Again, there were five sessions, each containing two compound stimulus presentations during the 12 th and the 24 th minute of the session.

The other three groups were treated exactly the same, except that they were returned to their cages for a 3-week retention interval between the first and the second acquisition phases. During this 3 -week period, these groups were maintained at $80 \%$ of their free-feeding weights.

On the day following the second acquisition phase, all six groups were given extinction trials to the previously used light. No shock was used in the extinction test phase, but water was still available on a VI 1-min schedule of reinforcement. Each rat was exposed to two 1-min light presentations during one extinction session. 


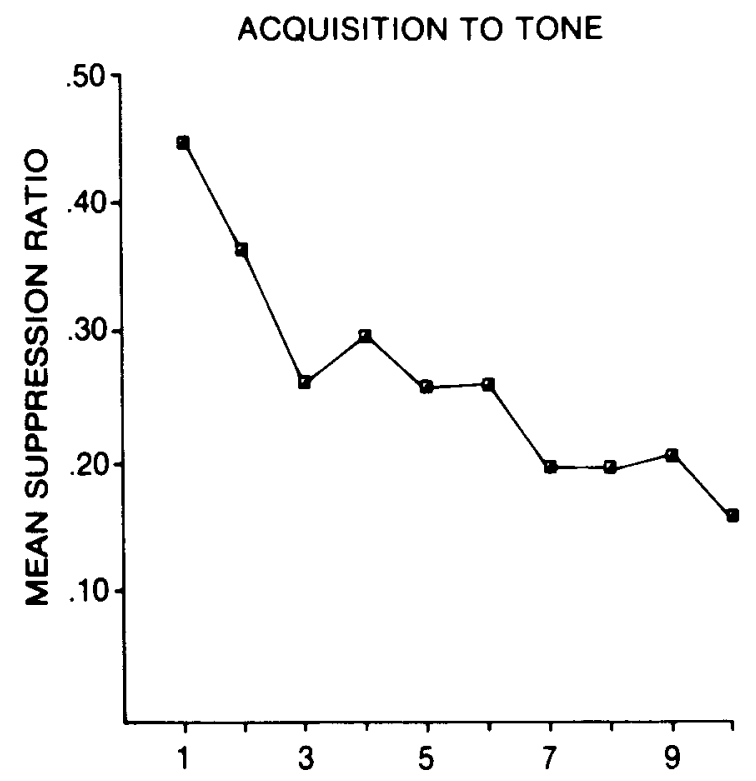

\section{ACQUISITION TO LIGHT-TONE COMPOUND FOLLOWING RETENTION INTERVAL}

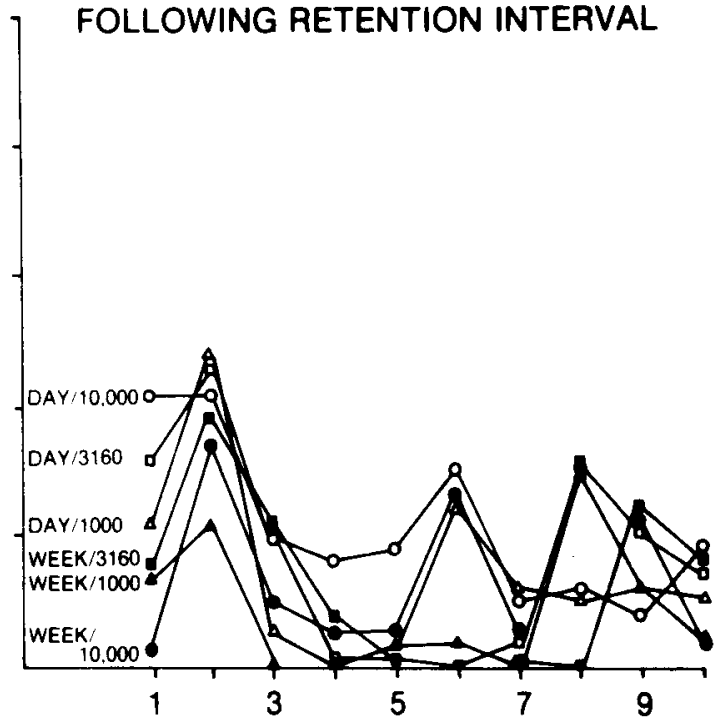

TRIALS

Figure 1. Mean suppression ratios for acquisition to the tone (left panel) and to the light-tone compound (right panel). The data are collapsed over groups during the first phase since there were no group effects, nor did the groups receive differential treatment during this phase.

\section{RESULTS}

Suppression ratios were calculated for each stimulus presentation. The ratios were of the form $A /(A+B)$, where $A$ is the rate of barpressing during the $C S$ presentation and $\mathrm{B}$ is the rate of barpressing during the minute immediately prior to the CS presentation. A score of .50 indicated relatively little suppression, and a score of 0.0 occurred under conditions of complete suppression (Annau \& Kamin, 1961).

Figure 1 displays the mean suppression ratios to the tone during the initial acquisition period and to the light-tone compound during the subsequent acquisition phase. For the first acquisition phase, in Figure 1, the mean suppression of all six groups is reported since all groups received identical treatment. An analysis of variance was performed on the suppression scores of the six groups during this acquisition period and revealed no differences among the groups $(F<1)$. As mentioned previously, rats were assigned to one of six groups at the end of this training with the intent of minimizing any differences. There was, however, a significant effect of trials $[F(9,216)=13.357, p<.001]$, indicating the acquisition of the suppression response. During suppression training to the tone, the mean pre-CS rates were 23.6, 20.5, 22.5, 18.6, 17.1, and 19.2 responses $/ \mathrm{min}$ for Groups Day/1,000, Day/3,160, Day/10,000, $\mathrm{Week} / 1,000$, Week $/ 3,160$, and Week $/ 10,000$, respectively $(F<1)$. These data suggest that the above analysis of ratio scores is uncomplicated by differences in baseline rates of barpressing.
Another analysis of variance was performed on the suppression scores of the six groups for the acquisition to the 10 light-tone trials. There were no retention interval or stimulus effects (Fs $<1$ ), but the effect of trials was again significant $[F(9,216)=$ $7.987, \mathrm{p}<.001]$. The mean suppression ratios for each of the six groups during the light-tone trials are reported in Figure 1. Pairwise comparisons of the first compound trial revealed no differences among the groups $(p>.05)$. That is, all the rats continued to suppress responding to the tone regardless of whether the frequency of the tone was changed and a light was added or just the light was added to the original tone. There was, however, a significant interval effect on this first compound trial $(p<.05)$, indicating that the 1-day-interval groups displayed less suppression to their respective compound stimuli than did the 3-week-interval groups. During acquisition to the light-tone stimulus, the mean pre-CS rates were $26.7,25.2,28.6,23.5,21.2$, and 21.2 responses/min for Groups Day/1,000, Day/3,160, Day $/ 10,000$, Week $/ 1,000$, Week $/ 3,160$, Week $/ 10,000$, respectively. There were no interval $[\mathrm{F}(1,24)=$ $2.816, \mathrm{p}<.10$ ], stimulus, or Interval by Stimulus interaction effects $(F s<1)$, which suggests that the above analysis of ratio scores is uncomplicated by baseline rate differences.

In order to examine the change in suppression from compound training to testing and to demonstrate the various treatment effects, an analysis of variance was performed on the six groups for the last light-tone trial and the light-only extinction trials. 
There was no effect of interval $[F(1,24)=6.814$, $\mathrm{p}<.14]$, but the Interval by Stimulus interaction was significant $[F(2,24)=4.014, p<.03]$ and the stimulus effect approached significance $[F(2,24)=$ 2.676, $p<.0878$ ]. Also, the effect of trials was significant $[F(2,48)=56.453, p<.001]$, as was the Interval by Stimulus by Trials interaction $[\mathrm{F}(4,48)$ $=2.609, \mathrm{p}<.05]$. The performance of each group on the last reinforced trial of the second acquisition phase and the light-only test trials is shown in Table 1. Pairwise comparisons were used to examine the differences in suppression between the last reinforced light-tone trial and the first extinction lightonly test trial. There was no change in suppression on this transitional trial for the Day/1,000 and the Day $/ 10,000$ groups. However, there was a significant change in suppression for the Day $/ 3,160$ groups and all the 3-week retention interval groups (ps $<.01$ ). This change was from relatively complete suppression at the end of the light-tone acquisition period to relatively little suppression on the light-only test trials.

A subsequent analysis of variance was performed on the extinction trials for all six groups. As evident in Table 1, both the Day/1,000 and the Day/10,000 groups showed more suppression on the test trials than the other four groups. This was confirmed by a significant effect of interval $[F(1,24)=12.827$, $\mathrm{p}<.0018]$ and Interval by Stimulus interaction $[F(2,24)=7.829, p<.0027]$. The effect of stimulus only approached significance $[F(2,24)=3.199, p<$ .0574]. Individual comparisons on the interaction revealed that both the Day/1,000 and the Day/10,000 groups showed significantly more suppression than the other four groups (ps $<.01$ ) over both test trials. There were no significant differences between these two groups or among the other four groups on these extinction trials. During testing, the mean pre-CS rates were $24.7,25.4,27.7,28.8,25.0$, and 25.9 responses/min for Groups Day/1,000, Day $/ 3,160$, Day $/ 10,000$, Week $/ 1,000$, Week $/ 3,160$, Week $/ 10,000$, respectively. There were no interval, stimulus, or Interval by Stimulus interaction effects $(\mathrm{Fs}<1)$.

\section{DISCUSSION}

These results are consistent with previous findings indicating relatively complete retention of CER over long delays. The groups trained after a 3-week interval with the original tone as part of the compound stimulus showed no change in suppression from the last tone trial to the first light-tone trial. Also, no forgetting was indicated in this group since blocking to the light was evident during testing. The present results also replicate Kamin's (1968) original finding of the blocking effect. The Day/3,160 group received the standard blocking treatment, and the rats in this
Table 1

Ratio Scores

\begin{tabular}{|c|c|c|c|}
\hline \multirow[b]{2}{*}{ Group } & \multirow{2}{*}{$\begin{array}{c}\text { Last Compound } \\
\text { Trial }\end{array}$} & \multicolumn{2}{|c|}{ Test Trial } \\
\hline & & 1 & 2 \\
\hline Day $/ 1,000$ & .05 & .10 & .24 \\
\hline Day $/ 3,160$ & .07 & .41 & .33 \\
\hline $\mathrm{Day} / 10,000$ & .09 & .22 & .19 \\
\hline Week $/ 1,000$ & .02 & .34 & .38 \\
\hline Week/3,160 & .08 & .31 & .33 \\
\hline Week $/ 10,000$ & .07 & .36 & .36 \\
\hline
\end{tabular}

group showed relatively little suppression to the added stimulus in the test trials, even though it had been paired with shock on 10 previous trials. More importantly, it appears that changing the original CS (tone) simultaneously with adding the second component (light) significantly attenuated the blocking effect in the 1-day retention interval groups.

Recently, a model of classical conditioning has been described (Rescorla \& Wagner, 1972) which states that a UCS is limited in the amount of conditioning it can support for a CS. One explanation for the blocking effect follows directly from this. Both of the elements of the compound stimulus compete for associative strength, and the extent to which one of the elements has received prior conditioning will limit the amount of successful conditioning to the other element. This model adequately accounts for the performance of the Day/3,160 group. Given this, the suppression performance of the Day/1,000 and the Day $/ 10,000$ groups during the extinction trials is reflecting the extent to which the rats in these groups responded to the second tone as novel. On the other hand, the Day/1,000 and the Day/10,000 groups did not show less suppression than the other four groups on the initial light-tone trial as required by this interpretation. Since there was no group that received only the light stimulus at this point in the experiment, it is not clear whether the first compound trial suppression was due to generalization from the orginal tone or to the unconditional effects of the light and/or the novel tone. However, other work in our lab suggests that the light and the tones have only minimal disruptive effects on barpressing performance. Also, the first compound-trial suppression performance of the Day/3,160 group is inconsistent with Kamin's (1968) work, which suggests that the addition of a second element disrupts suppression performance on the first compound trial. However, Mackintosh (1975, Experiment 1) has demonstrated the blocking effect using conditioned suppression of licking where the first compound-trial suppression was unchanged. Also, these data are not necessarily inconsistent with models that attribute blocking to selective attention to the previously learned element (Sutherland \& Mackintosh, 1971) or to learning that 
the added element is redundant (Mackintosh \& Turner, 1971).

Coulter's (Note 1) suggestion that certain attributes of a CS may be forgotten over time is supported by the results of the present experiment. All three groups with a 21-day retention interval showed little suppression to the added stimulus regardless of whether the tone used in the compound was the original or a novel component. The extinction performance of these groups did not differ significantly from the Day/3,160 group. That is, they responded to the added element just like the rats for which the compound stimulus contained a previously conditioned element. It appears that they responded to the compound as if it were the original CS. Thus, these data are also reminiscent of the Perkins and Weyant (1958) demonstration of increased generalization over time. Subsequent test trials revealed that no conditioning had accrued to the light. Also, this is the first report of blocking where both elements of the compound stimulus are novel. This finding is consistent with the notion that the frequency of the original CS was forgotten over time. The rats in the 1day groups, however, were able to retain the frequency of the original training tone over the short retention interval, as evidenced by their lack of blocking to the light when different tones were substituted in the compound.

It is possible that the Pavlovian conditioning experience is stored in separate memories (Rescorla, 1973), corresponding, perhaps, to the CS, the UCS, and the CS-UCS contingencies. These memories may be subject to different types of retention deficits or decay rates. If this is the case, it is apparent that at least certain characteristics of a CS that predicts shock are subject to forgetting, whereas the CS-UCS contingency (fear) is fairly resistent.

\section{REFERENCE NOTE}

1. Coulter, X. Long-term forgetting of conditioned emotional responses $(C E R)$. Paper presented at the annual meeting of the Eastern Psychological Association, Philadelphia, 1974.

\section{REFERENCES}

Annau, Z., \& Kamin. L. J. The conditioned emotional response as a function of the intensity of the US. Journal of Comparative and Physiological Psychology, 1961, 54, 428-432.
Campbell, B. A., \& Campbell, E. H. Retention and extinction of learned fear in infant and adult rats. Journal of Comparative and Physiological Psychology, 1962, 55, 1.8.

Campbell, B. A., \& Teghtsoonian, R. Electrical and behavioral effects of different types of shock stimuli on the rat. Journal of Comparative and Physiological Psychology, 1958, 51, 185-192.

Coulter, X., Collier, A. C., \& Campbell, B. A. Long-term retention of early Pavlovian fear conditioning in infant rats. Journal of Experimental Psychology: Animal Behavioral Processes, 1976, 2, 48-56.

Frieman, J. P., Warner, J., \& Riccio, D. C. Age differences in conditioning and generalization of fear in young and adult rats. Developmental Psychology, 1970, 3, 119-123.

Gleitman, H. \& \& Holmes, P. Retention of incompletely learned CER in rats. Psychonomic Science, 1967, 7, 19-20.

Hoffman, H. S., Selekman, W., \& Jensen, P. Stimulus aspects of aversive controls: Long-term effects of conditioned suppression procedures. Journal of the Experimental Analysis of Behavior, 1966, 9.659-662.

KAMIN, L. J. "Attention-like" processes in classical conditioning. In M. R. Jones (Ed.), Miami Symposium on the Prediction of Behavior: Aversive stimulation. Miami: University of Miami Press, 1968.

Mackintosh, N. J. Blocking of conditioned suppression: Role of the first compound trial. Jourmal of Experimental Psychology: Animal Behavioral Processes, 1975, 1, 335-345.

Mackintosh. N. J., \& Turner, C. Blocking as a function of novelty of CS and predictability of UCS. Quarterly Journal of Experimental Psychology, 1971, 23, 359-366.

Perkins, C. C.. \& Weyant, R. G. The interval between training and test trials as determiners of the slope of generalization gradients. Journal of Comparative and Physiological Psychology. 1958, 51, 596-600.

Rescorla, R. A. A model of Pavlovian conditioning. In U. S. Rusinov (Ed.), Mechanisms of formation and inhibition of conditional reflex. Moscow: "Nauka" Academy of Sciences of the USSR, 1973.

Rescorla, R. A., \& W Agner, A. R. A theory of Pavlovian conditioning: Variation in the effectiveness of reinforcement and nonreinforcement. In A. Black \& W. K. Prokasy (Eds.), Classical conditioning II. New York: Appleton-Century-Crofts, 1972.

Snedden, D. S., Spevack, A. A., \& Thompson, W. R. Conditioned and unconditioned suppression as a function of age in rats. Canadian Journal of Psychology, 1971, 25, 313-322.

SPEAR, N. E. Retrieval of memory in animals. Psychological Review, 1973, 80, 163-194.

Sutherland, M. S., \& Mackintosh, N. J. Mechanisms of animal discrimination learning. New York: Academic Press, 1971.

(Received for publication July 18, 1978; revision accepted August 27, 1978.) 